\title{
Variable stars from two surveys of microlensing in M31
}

\author{
Arlin P.S. Crotts ${ }^{1}$, Richard P. Boyle ${ }^{2}$, Christopher J. Corbally ${ }^{2}$, \\ and the MEGA Collaboration \\ ${ }^{1}$ Department of Astronomy, Columbia University, 550 West 120th \\ Street, New York, NY 10027, USA \\ ${ }^{2}$ Vatican Observatory Research Group, University of Arizona, Tucson, \\ AZ 85721, USA
}

\begin{abstract}
Recently we completed the VATT/Columbia survey monitoring a portion of M31 for microlensing and stellar variability, and report here on the microlensing result. Also, we have nearly completed a much larger survey (MEGA) covering most of the stars in M31, for which we have started cataloging many tens of thousands of Cepheids, Miras, eclipsing variables and other kinds of variable stars. The microlensing results from these two surveys hint that a significant fraction of the dark matter in M31 is composed of stellar-mass objects in the halo.
\end{abstract}

\section{Surveys: description, analysis and results}

The composition of dark matter in disk galaxies remains a mystery. The MACHO survey revealed a microlensing rate unanticipated in the context of the visible stellar population alone, that may compose a significant part of the dark matter halo (Alcock et al. 2000), consistent with only upper limits from EROS (Afonso et al. 2003). A decade ago we proposed that M31 offers a favorable alternative for probing the halo dark matter in disk galaxies, using microlensing of stars in M31 itself for lenses primarily in M31. Such a signal could be easily distinguished in terms of an asymmetry in microlensing rate across the face of M31 (Crotts 1992). This required developing techniques for the application of image subtraction to a time series of images (Tomaney \& Crotts 1996), which led to the first candidate microlensing events in M31 (Crotts \& Tomaney 1996). Several years of such observations were required both to amass sufficient lensing events for a statistically meaningful sample and to cull out variable stars by extending the baseline. M31 microlensing surveys have also been conducted by the AGAPE groups (Calchi Novati et al. 2002), and the survey reported here has been extended (de Jong et al. 2003). Together our two surveys hint that much of the dark halo might be composed of stellar-mass lensing objects.

The VATT/Columbia survey consisted of over 200 epochs of observation on three telescopes (the 1.8-m Vatican Advanced Technology Telescope (VATT) on Mt. Graham, Arizona; the MDM 1.3-m McGraw-Hill telescope on Kitt Peak, Arizona, with additional data from the Isaac Newton Telescope (INT) on La Palma, Canary Islands). Data were taken in $R$ and $I$ bands in two fields on either side of M31 along its minor axis placed so as to maximize the effects of 
halo microlensing asymmetry. We summarize the results of this survey below, and they are detailed in Uglesich et al. (2003).

Four microlensing events (an example of which is shown in Fig. 1) from VATT/Columbia survive a series of seven filter criteria in event $S / N$, sampling, and intrinsic variable rejection based on color and baseline variability. Our selection criteria allow us to compute an explicit detection efficiency and compare it to theoretical event rates based on Baltz et al. (2003). A maximum likelihood calculation yields contours in halo fraction $f_{b}$ and component mass $m$. If we marginalize over $f_{b}$ and $m$ in turn, we determine a measurement for each parameter separately to be microlensing halo mass fraction of $f_{b}=0.29_{-0.13}^{+0.30}$ of the total dark matter, and a lensing component mass $m$ between 0.02 and $1.5 \mathrm{M}_{\odot}$ $\left(1 \sigma\right.$ limits, with a most-favored value of $\left.0.53 \mathrm{M}_{\odot}\right)$. This compares with the MACHO survey (Alcock et al. 2000) that detects 13-17 events towards the LMC when only $\sim 2-4$ events would have been expected due to known stellar populations. The maximum likelihood halo fraction is $f_{b}=20 \%(8 \%-50 \%$ within a 95\% confidence interval). The M31 halo microlensing fraction $f_{b}$ we find is consistent with that seen by MACHO towards the LMC, but is only marginally inconsistent with no halo. The first results from MEGA (de Jong et al. 2003) also show a marginally significant far-side surplus asymmetry. While this other work does not estimate $f_{b}$, such an asymmetry is a nearly unique marker of a dark matter halo of microlensing objects (or a stellar spheroidal distribution of extreme mass and mass/light ratio, which approaches the same thing).

The MEGA survey ("Microlensing Exploration of the Galaxy and Andromeda") is being completed on five telescopes with wide field CCD imagers: KPNO 4-m/MOSAIC, INT/WFC, both MDM telescopes (2.4-m and 1.3-m) with the 8K, and lesser amounts of data from CFHT/12K and Subaru/ SuprimeCam. Over 300 epochs of observation are complete, and data from the INT and KPNO are analyzed. See Crotts et al. (2001) and de Jong et al. (2003) for details. MEGA has already isolated over 30000 Mira-like variables and a comparable number of Cepheids. In addition there are many thousands of other variable stars, in particular many eclipsing variables. While most of the data from MEGA still must be included in these light curves, some samples are shown in Fig. 2. Ultimately, time coverage and intensity will amount to about 15 times the number of points shown here (KPNO 4-m points only), and many more variable stars will be isolated from our images, covering most of the stellar population of M31. We expect these results to be available in 2004 .

\section{References}

Afonso, C., et al. 2003, A\&A, 400, 951

Alcock, C., et al. 2000, ApJ, 542, 281

Baltz, E.A., Gyuk, G., Crotts, A.P.S. 2003, ApJ, 582, 530

Calchi Novati, S., et al. 2002, A\&A, 381, 848

Crotts, A.P.S. 1992, ApJ, 399, L43

Crotts, A.P.S., Tomaney, A.B.T. 1996, ApJ, 473, L87

Crotts, A., et al. 2001, in ASP Conf. Ser., Vol. 239, Microlensing 2000, eds J. Menzies \& P. Sackett (San Francisco: ASP), 318 
de Jong, J.T.A., et al. 2003, A\&A, in press (astro-ph/0307072)

Tomaney, A.B.T., Crotts, A.P.S. 1996, AJ, 112, 2872

Uglesich, R.R., Crotts, A.P.S., Baltz, E.A., de Jong, J., Boyle, R.P., Corbally, C.J. 2003, ApJ, submitted

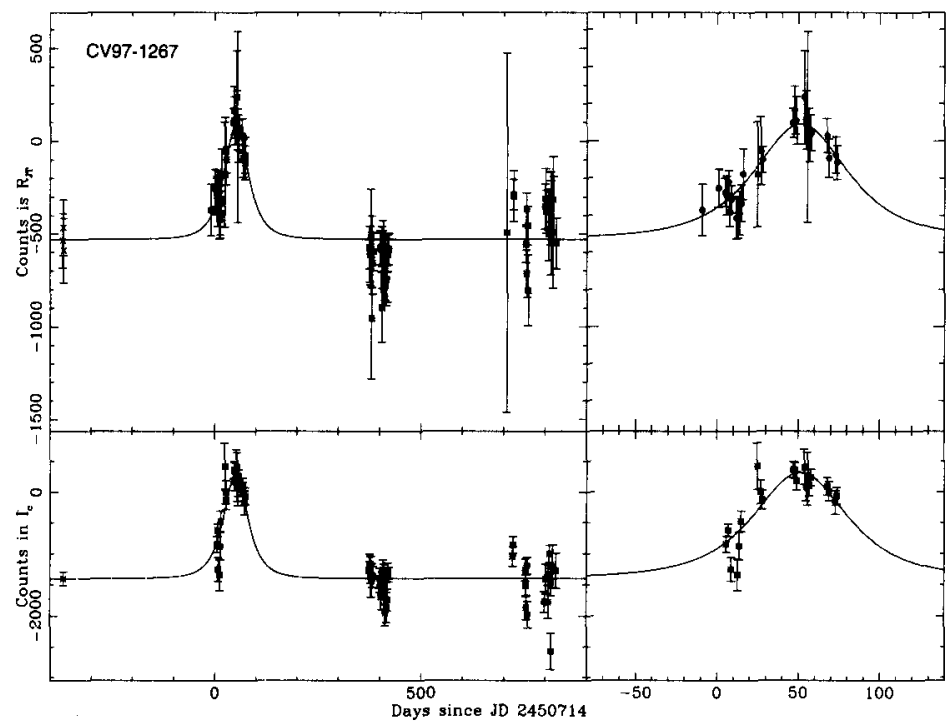

Figure 1. An example of a candidate light curve from the VATT/Columbia survey, plotted in ADU counts in the $R$ and $I$ bands as a function of Julian date. Points used in the Paczynski fits (from the MDM 1.3-meter) are indicated by the solid symbols; data from the VATT (first season) and INT (last season) are indicated by small diagonal crosses. 

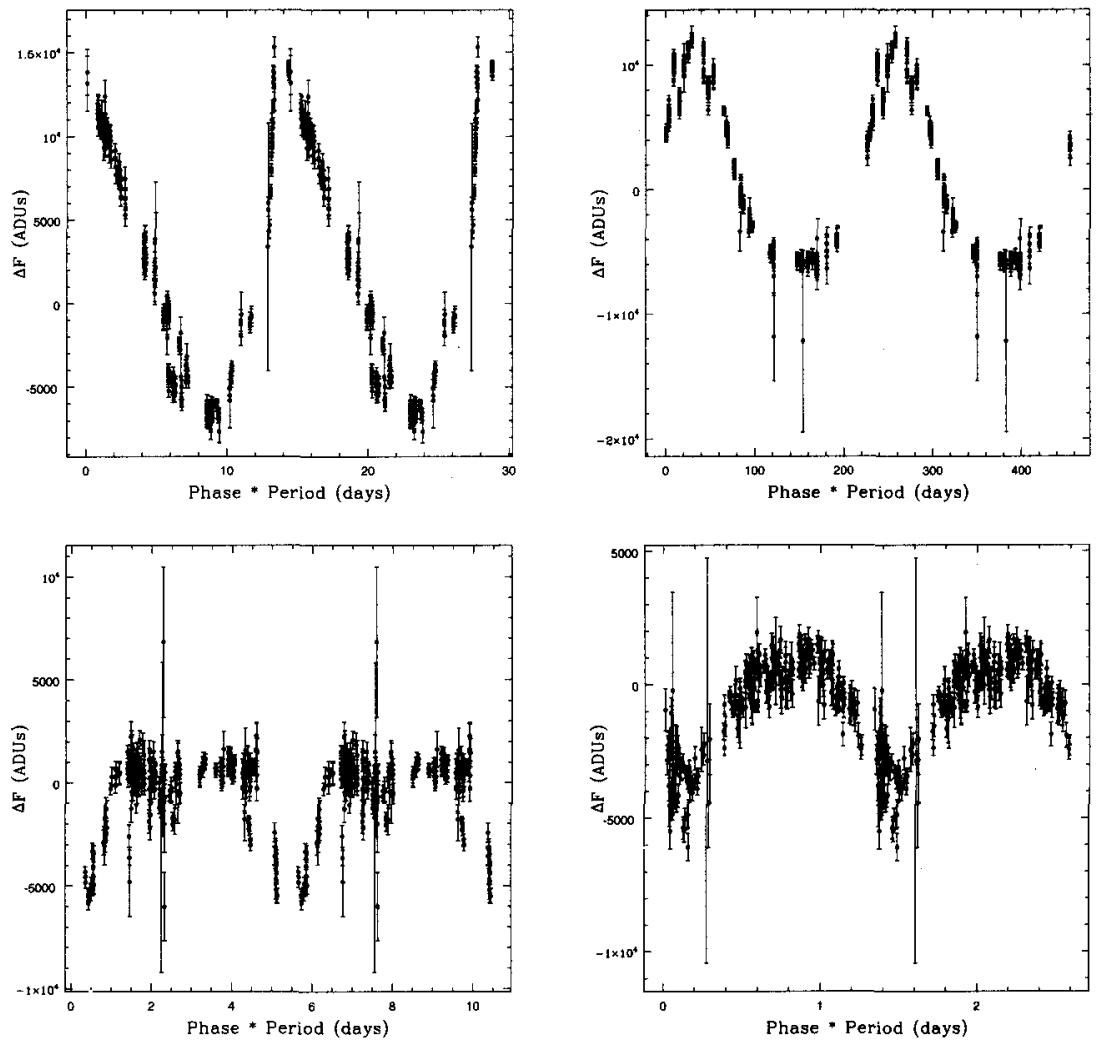

Figure 2. Some variable star light curves from the KPNO $4 \mathrm{~m} /$ MOSAIC sample of MEGA, including a Cepheid, a Mira-like variable, an eclipsing variable and a possible contact binary. These curves contain only about $6 \%$ of the light curve points that we will publish in our final catalog. 